\title{
Comment on Wei et al.: Comparison of artificial total disc replacement versus fusion for lumbar degenerative disc disease: a meta-analysis of randomized controlled trials
}

\author{
Guoping Liao • Fangwen Deng • Xinsheng Yin • Jingming Lin
}

Received: 1 June 2013 / Accepted: 17 June 2013 / Published online: 20 July 2013

(C) Springer-Verlag Berlin Heidelberg 2013

\section{Dear Editor,}

We read with great interest the recent paper "Comparison of artificial total disc replacement versus fusion for lumbar degenerative disc disease: a meta-analysis of randomized controlled trials" published online in the May 2013 issue of International Orthopaedics by Wei et al. [1]. The authors performed a meta-analysis of six randomized controlled trials to compare artificial total disc replacement with fusion for treatment of lumbar degenerative disc disease. It is an interesting study. Nevertheless, we have several methodological issues which we would like to communicate to the authors.

1. With respect to the assessment of study quality, two investigators independently evaluated the quality of each study. However, we would like to know how problems were if there were discrepancies between the two authors.

2. In the article, the authors used the odds ratio (OR) to report the risk value rather than relative risk (RR). As there are six randomized controlled trials included, RR can report the real risk value better than OR. Therefore, we suggest that RR should be used to report the risk value in this article.

G. Liao $\cdot$ F. Deng $\cdot$ X. Yin

Changning Hospital of Traditional Chinese Medicine, Changning

421500, Hunan Province, People's Republic of China

J. Lin $(\bowtie)$

Department of Pharmacy, Zhujiang Hospital, Southern Medical University, Guangzhou 510282, People's Republic of China e-mail: linjm1231@21cn.com
3. In the results section, the authors used the mean difference (MD) as summary statistic rather than standardised mean difference (SMD) for continuous outcomes, while they write a "standardised mean difference" in the results. We suggest that "standardised mean difference" should be replaced by "mean difference". However, effect sizes expressed as SMDs are a useful method to compare the effect of an intervention across studies when different measures (such as visual analogue scale and Oswestry disability index) are used.

4. In the results, the authors used a fixed effects model to pool the data in evaluating complication and reoperation rate for TDR and fusion groups at two years, while they write a "random effects pooled OR" in the results. We suggest that "random effects pooled OR" should be replaced by "fixed effects pooled OR". Meanwhile, they used a fixed effects model to pool the data in evaluating complication and reoperation rate, while the results showed a large amount of heterogeneity $\left(I^{2}=63 \%\right.$ or $\left.I^{2}=51 \%\right)$. In this case, why not make a sensitivity analysis or use a random effects model to pool the data?

5. It is not appropriate that a summary mean difference (MD) estimate with corresponding $95 \%$ CIs was derived using the method of inverse variance (IV) with the assumptions of a random-effects model. However, studies should be combined by using the DerSimonian and Laird random-effects model, which considers both within- and between-study variations [2].

6. There are different types in either disc or fusion for lumbar degenerative disc disease, which would bring different results. If possible, we suggest that a meta-analysis of artificial total disc replacement 
with different disc versus fusion with different surgical approaches for lumbar degenerative disc disease should be conducted.

In conclusion, the results of the study by Wei et al. should be interpreted with caution. To reach a definitive conclusion, further studies based on more carefully and scientifically designed RCTs with large samples and long-term follow-up are still needed to assess artificial total disc replacement versus fusion for lumbar degenerative disc disease. We believe that our remarks will contribute to more accurate evaluation of the results presented by Wei et al.

\section{References}

1. Wei J, Song Y, Sun L, Lv C (2013) Comparison of artificial total disc replacement versus fusion for lumbar degenerative disc disease: a meta-analysis of randomized controlled trials. Int Orthop. doi:10. 1007/s00264-013-1883-8

2. DerSimonian R, Laird N (1986) Meta-analysis in clinical trials. Control Clin Trials 7:177-88 